\title{
THEORETICAL ANALYSIS OF THE RELATIONSHIP BETWEEN THE ELECTRONIC STRUCTURE AND ITS INHIBITORY ACTION IN THE P2X7R RECEPTOR OF A SERIES OF 2-HYDROXY-1,4-NAPHTHOQUINONES DERIVATIVES
}

\author{
${ }^{1}$ CARLOS SOLOAGA ARDILES ${ }^{*}{ }^{2}$ JOSÉ CÁRCAMO VEGA \\ ${ }^{1}$ Departamento de Química, Universidad de Tarapacá, Av. General Velásquez 1775, P.O. Box 7-D Arica, Chile. \\ ${ }^{2}$ Laboratorio de Análisis e Investigaciones Arqueométricas (LAIA), Instituto de Alta Investigación (IAI), Universidad de Tarapacá, \\ Av. General Velásquez 1775, P.O. Box 7-D, Arica, Chile.
}

\begin{abstract}
In this work, a theoretical study of the relationship between the electronic structure and the activity of the P2X7R receptor from 2-hydroxy-1,4-naphthoquinone derivatives, using the KPG method, was performed. The electronic structure of the molecules was calculated at level B3LYP / 6-31G (d, p) with full geometry optimization. A statistically significant equation was obtained by relating the variation of biological activity with the variation of a set of indices of local atomic reactivity. Based on the analysis of the results, a two-dimensional pharmacophore was proposed.
\end{abstract}

Key words: P2X7R receptor, 2-hydoxy-1,4-naphthaquinones derivatives, KPG method (QSAR).

\section{INTRODUCTION}

The P2X7 receptor is a ligand-dependent ion channel that belongs to the type 2 purinergic receptor family (P2)[1]. The $\mathrm{P} 2$ receptor family comprises the P2Y G protein-coupled receptors (P2Y1, 2, 4, 6, 11-14) and the P2X (P2X1-7) receptors, which are ion channels regulated by ligand. $\mathrm{P} 2 \mathrm{X} 7$ is the receptor subtype most widely studied from an immunological perspective[2, 3]. Its activity can be found in a limited number of cell types, but it is easily detectable in hemopoietic lineage cells that include macrophages, microglia, and specific lymphocytes, and mediates the influx of $\mathrm{Ca}^{2+}$ and $\mathrm{Na}^{+}$, as well as the release of pro-inflammatory cytokines[4, 5]. The $\mathrm{P} 2 \mathrm{X} 7$ receptor can initiate large-scale intracellular ATP release through its intrinsic pore-forming ability, therefore, potentiating purinergic signaling and inflammation[6, 7]. Since many diseases involve inflammation and immune responses, and P2X7R regulates inflammation, there has been a recent interest in the pathophysiological functions of P2X7R and the potential of P2X7R antagonists to treat a variety of diseases $[8,9]$. These include neurodegenerative diseases, psychiatric disorders, epilepsy and a range of diseases of peripheral organs, including cardiovascular, respiratory, kidney, liver, bladder, skin and loco-motor systems[10, 11].

P2X7R inhibiting substances have been used as therapeutic agents[12, 13]. However, clinical trials in patients with rheumatoid arthritis using AZD-9056 and CE-224535 showed no improvement in the disease compared to the current clinical treatment. Thus, arises the need to try new selective inhibitors for this receptor. Consequently, the use of quinones, which is found in nature, could be a good response to this need[13, 14]. For which it is essential to know the type of molecular interaction using a theoretical study of the electronic structure of the 2-hydroxy-1,4-naphthoninone derivatives (Table 2) and their possible interaction with the $\mathrm{P} 2 \mathrm{X} 7 \mathrm{R}$ receptor, to obtain information, from this biological process. 2-hydroxy-1,4-naphthoninone derivatives have recently been proposed as potent, selective antagonists of $\mathrm{P} 2 \mathrm{X} 7$ activation, which allows carrying out clinical trials in inflammatory disorders and mediated by the immune system $[15,16]$.

The 1,4-naphthoquinone structure is found in a large number of compounds of natural origin and is associated with various biological properties [17]. In most cases, the biological activity of naphthoquinones has been related to their oxidation-reduction and acid-base properties, which can be modulated by synthetically modifying the substituents attached to the 1,4-naphthoquinone ring (Fig 1). The synthesis of new 1,4-naphthoquinone derivatives is of particular importance since these compounds show essential activities as antiparasitic, antibacterial, antifungal and anticancer agents $[18,19]$

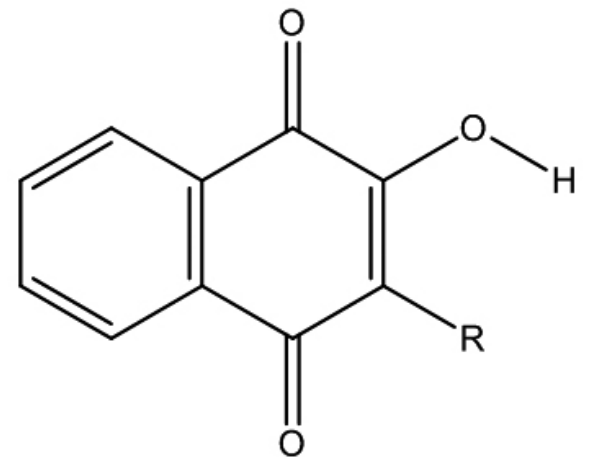

Figure 1. General structure of 2-Hydroxy-1,4 naphthoquinone

A theoretical study based on quantum chemistry, on the pharmacological effects in the interaction of the 1, 4-naphthoquinone derivatives with the $\mathrm{P} 2 \mathrm{RX} 7$ receptor, is of interest, since it allows knowing the biological process, involved in said interaction at atomic level. Until now, no theoretical studies have been reported on pharmacological effects on the interaction between derivatives of 1,4.naphthoquinone with the $\mathrm{P} 2 \mathrm{RX} 7$ receptor.

Method, models and calculations.

The method used in this work relates the variation of a biological activity, measured in vivo or in vitro, with the variation of the numerical values of a set of indexes of local atomic reactivity (LARI) [20]. The introduction of local atomic reactivity indices within the Hartree-Fock scheme plus the orientation parameter of the substituents has produced excellent results between electron structure relationships and biological activity for many different molecules and biological measurements. This method is known as KPG (Klopman-Peradejordi-Gómez). This QSAR method is characterized by using only parameters that come from quantum chemistry [21]. All this in order to make a theoretical prediction of the biological activity that allows the theoretical design of possible new drugs, avoiding going through the trial process and organic synthesis error [22].

In 1967, Klopman and Hudson presented a general perturbation model for chemical reactivity that includes ionic and unrestricted interactions only to $\pi$ electron $[23,24]$. In its model, the change of electronic energy, $\Delta \mathrm{E}$, associated with the interaction of atom $i$ of molecule $A$ with atom $j$ of molecule $B$ is given by [23] : 


$$
\Delta E=\sum_{p}\left[Q_{i} Q_{j} / R_{i j}+(1 / 2)\left(\beta_{i j}^{2}\right) \sum_{m} \sum_{n} F_{m i} F_{n j} /\left(E_{m}-E_{n}\right)-\left((1 / 2)\left(\beta_{i j}^{2}\right) \sum_{m} \sum_{n} F_{m i} F_{n j} /\left(E_{m}^{\prime}-E_{n}\right)\right]\right.
$$

A qualitative compression of the vast majority of chemical phenomena (but not all) is using a model based on the interaction between molecular orbitals. The type of drug-receptor interaction studied is weak and does not involve the formation of covalent bond.

The first term on the right side of Eq. Represents the electrostatic interaction between two atoms with net charge Qi and Qj. The following two terms introduce the interactions between the occupied and empty OMs of the drug with the OMs of the recipient. Note that the interactions are of the general type OM (busy) -OM (empty).

Where is the net charge of the atom I, $F_{m i}$ is the Fukui index of OM m of the atomi, $\beta_{i j}$ is the integral of resonance (supposedly be independent of the type of atomic orbitals (OA) because the $\mathrm{AB}$ complex does not involve covalent bonds) $E_{m}\left(E_{m}\right)$. Is the energy of m-sm occupide MO ( $m$ for the empty MOs) of the molecule A. $n$ and $n$ it refers to molecule B. The sum of $p$ reffers to all the pairs of átoms that interact. The first term on the right side of equation 1 represents the electrostatic interaction between the atom with net charges $Q_{i}$ and $Q_{j}$. The next two terms introduce the interactions between occupied $\mathrm{MO}$ of one molecule with the empty $\mathrm{MO}$ of the other molecule and vice versa. As this model represents the interaction energy regarding atom-atom.It was proposed that the constants of inhibitory velocity, affinity constant can be expressed as [25] :

$$
\log K_{i}^{I}=\text { cons } \tan t+\log K_{i}^{c}
$$

Where is the drug-receptor equilibrium constant. Now, considering the state of the thermodynamic stability and a 1:1 stoichiometry in the formation of the involved drug receptor:

$$
D_{i}+R \leftrightarrow D_{i} R
$$

$\underset{i}{D_{i}}$ is the drug, $R$ is the receptor and $D_{i} R$ is the complex drugten as:

According to statistical thermodynamics, the equilibrium constant is writ-

$$
K_{i}=\frac{Q_{D_{i} R}}{Q_{D_{i}} Q_{R}} \exp \left(-\Delta \varepsilon_{0}^{i} / K T\right)
$$

Where $Q_{D, R}, Q_{D}$ and $Q_{R}$, are the functions of partitioning the drugreceptor complex, $K$ is Boltzmann's constant and the absolute temperature. $\Delta \varepsilon_{0}$ is the energy difference of the ground state of the drug-receptor complex $\left(Q_{D_{i} R}\right)$ and the energies of the fundamental state of the drug $\left(Q_{D_{i}}\right)$ and the receptor $\left(Q_{R}\right)$ :

$$
\Delta \varepsilon_{0}^{i}=\varepsilon_{D_{i} R}-\left(\varepsilon_{D_{i}}+\varepsilon_{R}\right)
$$

Peradejordi et al. consider that the terms of the partition function and the solvation energy are constant. After some considerations and approximations, the linear equations are obtained:

$$
\log K_{i}^{I}=A+\sum_{P}\left\{a_{p} Q_{b, p}+b_{p} S_{b, p}^{E}+c_{p} S_{b, p}^{N}\right\} i=1,2, \ldots, n
$$

where ${ }_{E} A, a_{p}, b_{p}, \quad$, are constants to determine. $Q_{b, p}$ is the net charge. $S_{b, p}$ is the total atomic electrophilic super-localization of the atom p y $S_{b, p}^{N}$ is the total atomic nucleophilic atomic super location of the atom
P. Work continued on interaction energy from the drug site arriving at the following equation:

The following linear equation (Eq. 7) represents this method [26] . This equation is applicable drug-receptor interactions[27].

$p K_{i}=a+\sum_{j}\left[e_{j} Q_{j}+f_{j} S_{j}^{E}+s_{j} S_{j}^{N}\right]+$

$\sum_{j} \sum_{m}\left[h_{j}(m) F_{j}(m)+x_{j}(m) S_{j}^{E}(m)\right]+\sum_{j} \sum_{m^{\prime}}\left[r_{j}\left(m^{\prime}\right)+t_{j}\left(m^{\prime}\right) S_{j}^{N}\left(m^{\prime}\right)\right]+$
$+\sum_{j}\left[g_{j} \mu_{j}+k_{j} \eta_{j}+o_{j} \omega_{j}+z_{j} \varsigma_{j}+w_{j} Q_{j}^{\max }\right]+\sum_{B=1}^{W} O_{B}$

Where $p K_{i}$ is the logarithm of the constant (K $\mathrm{KC} 50$, IC50, etc.). $Q_{i}$ is the net charge of atom j, $S_{j}^{E}$ y $S^{N}$ are, respectively, the total electrophilic and nucleophilic superdelocalizability of Fukui. These are defined as the summation over all the occupied OMs (electrophilic) of the atom $\mathrm{j}$, and the summation over all the empty OMs (nucleophilic) of the atom j. $F_{2}\left(F_{,},\right)$is the Fukui index of the occupied molecular orbital (m) and empty molecular orbital (m,) respectively, located on the $\mathrm{j}$ atom. $S_{j}^{m}(\mathrm{~m})$ is the atomic electro-physical superlocalization of $\mathrm{OM} m$ located at atom $\mathrm{j}$, etc. In equation 1 , the terms enclosed in brackets contain a series of local reactivity indices, obtained in the framework of the Hartree-Fock models LCAO-MO and DFT [28]. Within indexes of local atomic reactivity can be mentioned: it is the local atomic hardness of the atom $\mathrm{j}, \mu_{j}$ is the local atomic electronic chemical potential of the atom $\mathrm{j} \omega_{j}$, is the local atomic electrophilicity of the atom $\mathrm{j}, \boldsymbol{\zeta}_{j}$, is the local atomic softness of the atom $\mathrm{j} Q_{j}^{\max }$ and is the maximum amount of electronic charge that the atom $\mathrm{j}$ can accept from another site, $\eta_{j}$ is the local atomic hardness of the atom $j$. The other terms of equation 1 are constant to determine. These indexes of local reactivity are associated with local molecular orbitals: HOMO $\mathrm{j} *$ is defined as the highest occupied local molecular orbital, while LUMO $\mathrm{j}$ *, is the lowest occupied empty local molecular orbital $[25,29,30]$.

$$
\log \left[(A B C)^{-1 / 2}\right]=\sum_{i} \sum_{t} m_{i, t} R_{i, t}^{2}=\sum_{t} O_{t}
$$

The summation runs for the different substituents of the molecule (Eq. 8), where $m_{i, t}$ is the mass of the atom i-th corresponding to the substituent $\mathrm{t}$-th and $R_{i, t}^{2}$ is the distance at which the atom of the substituent is bound. The physical interpretation of the orientation parameter represents the fraction of molecules that reach the correct orientation to interact with the receptor [31].

The HFR method has provided, over time, a set of local reactivity indices that have shown their usefulness in fields ranging from chemical reactivity to studies of structure-activity relationships [32]. Two types of data are interesting: a matrix that contains the LCAO coefficients and another that includes the eigenvalues (the OM energies). In general, by using the LCAO coefficient matrices and the coating matrix we can assign electronic populations (that is, a whole or fractional number of electrons) to each atomic orbital of the base used and to each OM. This makes the population analysis of Mulliken fundamental to obtain these populations and calculations of eigenvalues of the energy of molecular orbitals [21,33].

The idea is from the new indexes of local atómic reactivity. It can be sumarized in the following figure $1[26]$ :

Where the circles represent populations of electrons. As shown, there are higher OMs occupied as (HOMO-1)" and (HOMO-2)* with non-zero electronic populations, as in the case of the lowest voids (LUMO+1)* and (LUMO+2)* It is also possible to obtain a (HOMO)* local or (LUMO) local when these coincide or not with the respective OMs. When the HOMO and the occupied OM that lie beneath it are energetically close, it is necessary to include them both within an index of reactivity $[34,35]$. In Figure 2 , the arrows indicate the local atomic hardness as an example. Within this scheme, a hard atom resists the 
exchange of electrons with the atoms of the other molecule. Thus, the highest occupied local molecular orbitals and the lowest empty local molecular orbitals, different from the molecular HOMO and LUMO, with non-zero electronic populations, are important in the atom-atom interactions, mainly in the weak interactions.
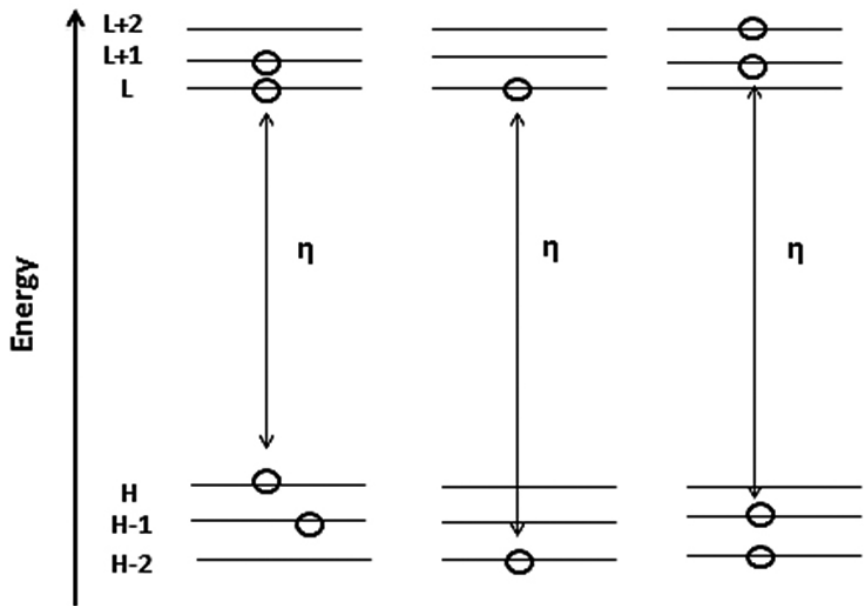

Figure 2. Diagram of molecular orbitals for three different atoms of the same molecule.

The new local atomic reactivity indexes (LARIs) of Eq. 7 are defines in the following manner:

Local atomic electronic chemical potential:

$$
\mu_{i}=\left(\varepsilon_{\text {HOMO }^{*}, i}+\varepsilon_{\text {LUMO }^{*}, i}\right) / 2
$$

Where $\varepsilon$ is the energy of the local molecular orbital of the atom $\mathrm{i}$.

Local atomic hardness:

$$
\eta_{i}=\left(\varepsilon_{\text {HOMO }_{, i}^{*}}-\varepsilon_{L U M O^{*}, i}\right)
$$

Local electrophilic superdelocalizability of the HOMO* of atom $\mathrm{i}$ and local nucleophilic superdelocalizability of the LUMO* of atom i:

$$
\begin{gathered}
S_{i}^{E^{*}}=\frac{F_{i, H O M O^{*}}}{\varepsilon_{\text {HOMO }^{*}}} \\
S_{i}^{N^{*}}=\frac{F_{i, L U M O^{*}}}{\varepsilon_{\text {LUMO }}}
\end{gathered}
$$

Where $F$ is the Fukui index of the atom i.

Local atomic softness of the atom $\mathrm{i}$ :

$$
S_{i}=\frac{1}{\eta_{i}}
$$

Where $\eta_{i}$ is the hardness of the atom i.

Local atomic electrophilicity of atom i:

$$
\omega_{i}=\frac{\mu_{i}^{2}}{2 \eta_{i}}
$$

\begin{tabular}{|c|c|c|}
\hline$Q_{i}$ & $\begin{array}{l}\text { Net atomic charge of } \\
\text { atom i Electrostatic } \\
\text { interaction }\end{array}$ & Electrostatic interaction \\
\hline$S_{i}^{E}$ & $\begin{array}{c}\text { Total atomic } \\
\text { electrophilic } \\
\text { superdelocalizability } \\
\text { of atom i }\end{array}$ & $\begin{array}{l}\text { Total atomic electron- } \\
\text { donating } \\
\text { capacity of atom i } \\
\text { (MO-MO } \\
\text { interaction) }\end{array}$ \\
\hline$S_{i}^{N}$ & $\begin{array}{c}\text { Total atomic } \\
\text { nucleophilic } \\
\text { superdelocalizability } \\
\text { of atom i }\end{array}$ & $\begin{array}{l}\text { Total atomic electron- } \\
\text { accepting } \\
\text { capacity of atom i (MO- } \\
\text { MO interaction) }\end{array}$ \\
\hline$S_{i}^{E}(m)$ & $\begin{array}{c}\text { Orbital atomic } \\
\text { electrophilic } \\
\text { superdelocalizability } \\
\text { of atom } \\
\text { i and occupied MO m }\end{array}$ & $\begin{array}{c}\text { Electron-donating capacity } \\
\text { of atom i at occupied } \\
\text { MO m } \\
\text { (MO-MO interaction) }\end{array}$ \\
\hline$S_{i}^{N}\left(m^{\prime}\right)$ & $\begin{array}{c}\text { Orbital atomic } \\
\text { nucleophilic } \\
\text { superdelocalizability } \\
\text { of atom } \\
\text { i and empty MO m' }\end{array}$ & $\begin{array}{c}\text { Electron-accepting } \\
\text { capacity } \\
\text { of atom i at empty MO m' } \\
\text { (MO-MO interaction) }\end{array}$ \\
\hline$F_{i}$ & Fukui index of atom $\mathrm{i}$ & $\begin{array}{l}\text { Total electron population } \\
\text { of atom I (MO-MO } \\
\text { interaction) }\end{array}$ \\
\hline$F_{m i}$ & $\begin{array}{l}\text { Fukui index of atom } \\
\text { i and } \\
\text { empty MO m' }\end{array}$ & $\begin{array}{l}\text { Electron population of } \\
\text { empty MO m' at atom I } \\
\text { (MO-MO interaction) }\end{array}$ \\
\hline$\mu_{i}$ & $\begin{array}{c}\text { Local atomic } \\
\text { electronic } \\
\text { chemical potential of } \\
\text { atom } \mathrm{i}\end{array}$ & $\begin{array}{l}\text { Propensity of atom i to } \\
\text { gain or } \\
\text { lose electrons }\end{array}$ \\
\hline$\eta_{i}$ & $\begin{array}{c}\text { Local atomic } \\
\text { hardness of atom i }\end{array}$ & $\begin{array}{l}\text { Resistance of atom i to } \\
\text { exchange electrons with } \\
\text { a site }\end{array}$ \\
\hline$\zeta_{i}$ & $\begin{array}{l}\text { Local atomic softness } \\
\text { of atom i }\end{array}$ & The inverse of $\mu_{i}$ \\
\hline$\omega_{i}$ & $\begin{array}{l}\text { Local atomic } \\
\text { electrophilicity } \\
\text { of atom i }\end{array}$ & $\begin{array}{c}\text { Propensity of atom i to } \\
\text { receive } \\
\text { extra electronic charge } \\
\text { together with its resistance } \\
\text { to exchange charge with } \\
\text { a site }\end{array}$ \\
\hline$O^{\max }$ & $\begin{array}{l}\text { Maximal amount of } \\
\text { electronic } \\
\text { charge atom i may } \\
\text { receive }\end{array}$ & $\begin{array}{c}\text { Maximal amount of } \\
\text { electronic charge that atom } \\
\text { i may receive from a donor } \\
\text { site }\end{array}$ \\
\hline
\end{tabular}

The maximal amount of charge atom i may receive:

$$
Q_{i}^{\max }=-\frac{\mu_{i}}{\eta_{i}}
$$

The physical meaning of these indices is summarized in Table 1.

Table 1. Local Atomic Reactivity Indices and their physical meaning.

These local indexes should provide information of interest in at least two cases. Within a molecule, they serve to identify the potentially most reactive sites accurately. They also serve to analyze, within an atom-atom interaction model, what could be the possible nature of the atom (s) with which they interact. This is especially important when the nature of one of the systems that 
interact is not known (one could know part of the structure of the interaction pharmacophore) [26]. Thus, the importance of this method lies in facilitating the processes of drug synthesis.

Table 2. Select 1,4 Naphthoquinone and their P2X7R functional activity (IC50).

\begin{tabular}{|c|c|c|c|}
\hline Mol. & Mol. & R & Log (IC50) P2X7R \\
\hline 1 & AN-01 & Hydroxyl & 1,88 \\
\hline 2 & AN-02 & Bromine & $-0,15$ \\
\hline 3 & AN-04 & benzyl & 1,64 \\
\hline 4 & AN-12 & 4-metyl-bencyl & 1,91 \\
\hline 5 & AN-13 & 3-fluoryne-bencyl & 1,52 \\
\hline 6 & AN-14 & 4-methoxy-bencyl & 1,9 \\
\hline 7 & AN-15 & 3-methoxy-bencyl & 0,5 \\
\hline 8 & An-16 & 4-cyano-bencyl & 1,66 \\
\hline
\end{tabular}<smiles>[R]C1=C(O[2H])C(=O)c2ccccc2C1=O</smiles>

Figure 3. Numbering of the common skeleton.

In the common skeleton, of the general structure of 2-Hydroxy-1,4 naphthoquinone (Fig. 1), the rings $\mathrm{A}$ and $\mathrm{B}$ are represented, the oxygen atoms attached to ring $\mathrm{B}$ in positions 1,2 and 4 and the first atom of the substituents in position 15 was considered (fig. 3). One of the functions of a substituent is to modify the electronic structure of the general skeleton [36]. The Functional activity (IC50) which indicates how much of a drug or other particular substance is needed to halve a given biological process (or component of a process) [37]. In this work, the inhibitory action of the derivatives of the 1,4 naphthoquinone (Table 2) on the $\mathrm{P} 2 \mathrm{X} 7$ receptor $(\mathrm{P} 2 \mathrm{X} 7 \mathrm{R})$ is considered.

The optimization of the geometry of the molecules and the obtaining of the electronic structure was achieved using DFT at a level of theory B3LYP 6-31G $(\mathrm{d}, \mathrm{p})$ with the Gaussian 09 software. The D-CENT-QSAR software [38] was used to obtain the local reactivity indexes. Multiple linear regression analysis (LMRA) was applied to discover which local atomic properties would be involved in the variation of biological activity throughout the series. The log (IC50) is considered as the dependent variable (8 values de IC50) and the local atomic reactivity indices, as the independent variables.

Now we have to solve the set of simultaneous linear equations. In this work, 18 atoms are belonging to the common skeleton, for 20 indices of reactivity. For this case 361 molecules are needed to obtain these parameters, constant included. Since data with so many molecules are usually not reported, multiple linear regression analysis should be used to find the most statistically significant equation $[21,26,39]$. Here the regression analysis is used, not to see if there is any structure-activity relationship, but to find the best of them.

\section{RESULTS AND DISCUSSION}

The following results show that the pharmacophore would indicate that the ligand would be interacting with the receptor through the $\mathrm{C} 4, \mathrm{C} 6$ and $\mathrm{C} 8$, by means of local atomic reactivity indexes, which are now being discussed:

From the linear regression analysis, the following local reactivity indices were obtained for three atoms of the common skeleton. These results represent the specific sites of interaction of the pharmacophore with the receptor.
The best statistically significant equation obtained is the following:

$\log (I C 50)=61.15-0.83 S_{6}^{E}(H O M O)^{*}-246.13 F_{4}(H O M O-2)^{*}$

$-1.97 S_{8}^{E}(H O M O-2)^{*}-9.51 Q_{6}^{\max }$

with $\mathrm{n}=8, \mathrm{R}=0.99, \mathrm{R}^{2}=0.99$, adj. $\mathrm{R}^{2}=0.99, \mathrm{~F}(6,12)=2334(\mathrm{p}<0.000001)$ and a standard error of estimate of 0.02 . No outliers were detected, and no residuals fall outside the $\pm 2 \sigma$ limits.

Table 3. Squared correlation coefficients for the variables appearing in Eq. 16.

\begin{tabular}{|c|c|c|c|c|}
\hline & $S_{6}^{E}(\text { HOMO })^{*}$ & $F_{4}(\text { HOMO }-2)^{*}$ & $S_{8}^{E}(H O M O-2)^{*}$ & $Q_{6}^{\max }$ \\
\hline$S_{6}^{E}\left(\mathrm{HOMO}^{*}\right.$ & 1.00 & & & \\
\hline$F_{4}(\text { HOMO }-2)^{*}$ & 0.11 & 1.00 & & \\
\hline$S_{8}^{E}(H O M O-2)^{*}$ & 0.21 & 0.07 & 1.00 & \\
\hline$Q_{6}^{\max }$ & 0.41 & 0.03 & 0.02 & 1.00 \\
\hline
\end{tabular}

Table 4. Beta coefficients and $t$-test for significance of the coefficients in Eq. 16.

\begin{tabular}{|c|c|c|c|}
\hline Variable & Beta & t (3) & p-level \\
\hline$S_{6}^{E}(H O M O)^{*}$ & -1.13 & 52.21 & $<0.00001$ \\
\hline$F_{4}(H O M O-2)^{*}$ & -1.70 & -55.14 & $<0.00001$ \\
\hline$S_{8}^{E}(H O M O-2)^{*}$ & -1.14 & -55.45 & $<0.00001$ \\
\hline$Q_{6}^{\max }$ & -0.44 & -41.70 & $<0.00003$ \\
\hline
\end{tabular}

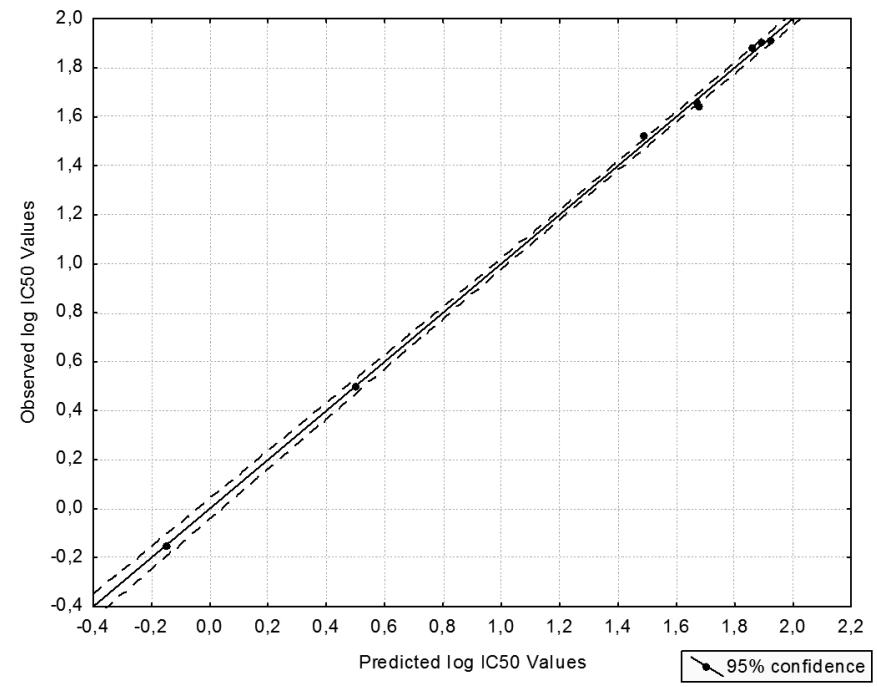

Figure 4: Plot of predicted vs. observed IC50 values (Eq. 16). Dashed lines denote the $95 \%$ confidence interval.

$S_{6}^{E}(H O M O)^{*}$ is the electrophilic superdelocalizability of the highest occupied local molecular orbital, localized on atom 9.

$F_{4}(H O M O-2)^{*}$ is the Fukui index (electron population) of the third highest occupied local molecular orbital, localized on atom 4. 
$S_{8}^{E}(H O M O-2)^{*}$ is the electrophilic superdelocalizability of the third highest occupied local molecular orbital, localized on atom 8

$Q_{6}^{\max }$ is the maximal amount of electronic charge that atom 6 may accept from another site.

Table 3 shows that the internal correlations between the independent variables are not significant. The statistical results related to the Eq. 16 show that this equation is statistically significant and corresponds to the variation of a group of four local atomic reactivity indices belonging to the general skeleton. These results explain approximately $99 \%$ of the variation of antagonist effects for the $\mathrm{P} 2 \mathrm{X} 7$ receptor (P2X7R). Also, figure 4 shows that there is a good correlation between observed values versus calculated values and that the points are within the $95 \%$ confidence interval [41].

Table 5. Nomenclature: Molecule (HOMO) / (HOMO-2) * (HOMO-1) * $(\mathrm{HOMO}) *-(\mathrm{LUMO}) *(\mathrm{LUMO}+1) *(\mathrm{LUMO}+2) *$

\begin{tabular}{|c|c|c|c|}
\hline Mol. & Atom 4 & Atom 6 & Atom 8 \\
\hline \multirow{2}{*}{$1(45)$} & $39 \sigma 40 \pi 45 \pi-$ & $41 \pi 43 \sigma 45 \pi-$ & $41 \pi 43 \sigma 44 \pi-$ \\
& $46 \pi 47 \pi 48 \pi$ & $46 \pi 47 \pi 48 \pi$ & $46 \pi 47 \pi 48 \pi$ \\
\hline \multirow{2}{*}{$2(62)$} & $57 \sigma 59 \pi 61 \pi-$ & $58 \pi 60 \pi 61 \pi-$ & $58 \pi--62 \pi-$ \\
& $63 \pi 64 \pi 65 \pi$ & $63 \pi 64 \pi 65 \pi$ & $63 \pi 64 \pi 65 \pi$ \\
\hline \multirow{2}{*}{$3(65)$} & $63 \sigma 64 \pi 65 \pi-$ & $62 \sigma 63 \sigma 64 \sigma-$ & $61 \pi--65 \pi-$ \\
& $66 \pi 67 \pi 68 \pi$ & $66 \pi 67 \pi 68 \pi$ & $66 \pi 67 \pi 68 \pi$ \\
\hline \multirow{2}{*}{$4(69)$} & $67 \sigma 68 \sigma 69 \pi-$ & $66 \sigma 67 \sigma 68 \sigma-$ & $--66 \sigma 69 \pi-$ \\
& $70 \pi 71 \pi 72 \sigma$ & $70 \pi 71 \pi 72 \pi$ & $70 \pi 71 \pi 72 \pi$ \\
\hline \multirow{2}{*}{$5(69)$} & $67 \sigma 68 \sigma 69 \sigma-$ & $66 \pi 67 \sigma 68 \pi-$ & $67 \sigma 68 \pi---$ \\
& $70 \pi 71 \pi 72 \pi$ & $70 \pi 71 \pi 72 \pi$ & $70 \pi 71 \pi--$ \\
\hline \multirow{2}{*}{$6(73)$} & $71 \pi 72 \sigma 73 \sigma-$ & $69 \sigma 70 \sigma 72 \sigma-$ & $69 \pi----$ \\
& $74 \pi 75 \pi 76 \sigma$ & $74 \pi 75 \pi 76 \pi$ & $74 \pi 75 \pi 76 \pi$ \\
\hline \multirow{2}{*}{$7(73)$} & $69 \pi 71 \sigma 72 \pi-$ & $67 \pi 70 \sigma 71 \sigma-$ & $69 \pi--$ \\
\hline \multirow{2}{*}{$8(71)$} & $74 \pi 75 \pi 76 \pi$ & $74 \pi 75 \pi 76 \pi$ & $72 \pi-74 \pi 75 \pi 76 \pi$ \\
\hline
\end{tabular}

The beta values (Table 4) show that the importance of the variables follows the following order:

$F_{4}(H O M O-2)^{*}>S_{8}^{E}(H O M O-2)^{*}>S_{6}^{E}(H O M O)^{*}>Q^{\max }$ The analysis of equation 16 shows that a high inhibitory capacity of the $\mathrm{P} 2 \mathrm{X} 7 \mathrm{R}$ receptor associated with high positive values for $F_{4}(H O M O-2)^{*}$. The atom 4 is a carbon of an aromatic ring to which it is attached to a ${ }^{4}$ zxygen atom. Table 5 shows that in all molecules, (HOMO-2) $*$ is of a nature $\sigma$ and $\pi$. It is suggested that atom 4 is interacting with an electron deficient center [43], through its third-highest occupied local molecular orbital. Although the (HOMO-2) * does not have a physical sense, the literature shows that the approach used is consistent with the published [26, 33, 44-47].

Low values for $S^{E}(H O M O-2)^{*}$ are associated with a high inhibitory capacity of the P2X7R receptor. For low values of this index, it is possible to decrease the electron population, generating a less reactive local MO, or decreasing the eigenvalue of local MO energy. The decrease of the energy of the molecular orbital can be obtained by merely eliminating the location of the real $(\mathrm{HOMO}-2)_{8} *$ so that an internal MO becomes the $(\mathrm{HOMO})_{8} *[42,48]$. The atom 8 is a carbon located in the aromatic ring A. According to table 5, its three highest local molecular orbitals occupied are of nature $\sigma$ and $\pi$. It is suggested that atom 8 is interacting with an electron-rich center through its first two occupied higher molecular orbitals $[23,49]$.

Low values for $S_{6}^{E}(H O M O)^{*}$ are related to a high inhibitory power of the P2X7R receptor. The atom 6 is a carbon of the aromatic ring A (fig. 3). Table 5 shows that it's (HOMO) $6 *$ is of nature $\sigma$ and $\pi$ and that the molecular (HOMO) coincide with the (HOMO) *. It is suggested that atom 6 is interacting with an electron-rich center of the receptor, though, its first highest occupied local orbital [50-52].

Equation 3 shows that a constant high inhibitory capacity of the P2X7R receptor is related to high values for $Q^{\max }$. How $\max$ is the value of the relationship between electronic chemical potential and hardness. A high value can be obtained, for example, by lowering the value of $\eta_{6}$ keeping constant . It is also possible to scroll down the value of on the energy axis $[48,53]$. High values for $Q_{6}^{\max }$ indicates that this atom (carbon of an aromatic ring) is interacting with a center rich in electrons and that it is a suitable acceptor of electrons. The above agrees perfectly with the result for $S_{6}^{E}(H O M O)^{*}$
These results are represented in figure 5 .

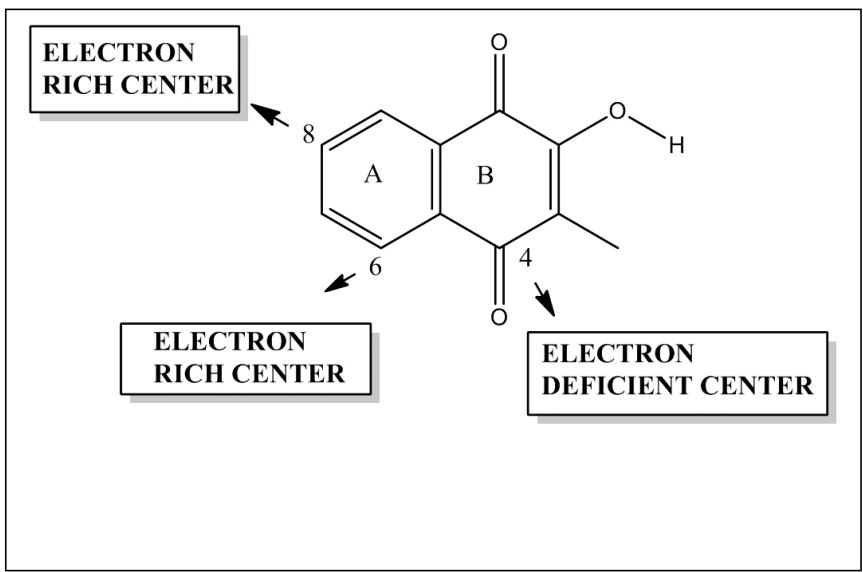

Figure 5. 2D pharmacophore for receptor binding P2X7R.

\section{CONCLUSIONS}

The use of MLRA to reveal the interactions that govern the inhibition of the $\mathrm{P} 2 \mathrm{X} 7 \mathrm{R}$ receptor by the 2-hydroxy-1,4-naphthoninone derivatives seems to be an appropriate methodology for this purpose. The results showed that there are specific zones of these derivatives are potentially involved in the biological process. The presence of the different substituents seems not to have direct participation in the interaction; their importance may be associated with the modification of the electronic structure of the common skeleton.

In summary, statistically significant relationships have been obtained between the electronic structure and the functional activity (IC50) for the P2X7R receptor. The corresponding pharmacophore was obtained. These results provide information that serves to a better understanding of this biological process.

\section{ACKNOWLEDGEMENTS}

This work was financially supported by project 11140262 from FONDECYT. The authors thank Dr. María Camarada, Director of the Center for Applied Nanotechnology, Faculty of Sciences Universidad Mayor for providing the means to carry out this study.

\section{REFERENCES}

1. Arulkumaran, N., R.J. Unwin, and F.W. Tam, A potential therapeutic role for P2X7 receptor (P2X7R) antagonists in the treatment of inflammatory diseases. Expert opinion on investigational drugs. Taylor \& Franci.; 20, 897, (2011).

2. Baggio, S.L.E., The P2X7 receptor in inflammatory diseases: Angel or demon? Front Pharmacol. 9, 52, (2018).

3. Franchi, L., et al., Differential requirement of P2X7 receptor and intracellular $\mathrm{K}+$ for caspase-1 activation induced by intracellular and extracellular bacteria. J. Bio. Chem., ASBMB. 282, 18810, (2007).

4. Burnstock, G. and G.E. Knight, The potential of P2X7 receptors as a therapeutic target, including inflammation and tumour progression Purinergic signalling. S Springer. 14, 18, (2018)

5. Di Virgilio, F., et al., Non-nucleotide agonists triggering P2X7 receptor activation and pore formation. Front. Pharmaco., Frontiers. 9, 39, (2018)

6. Safya, H., et al., Variations in Cellular Responses of Mouse T Cells to Adenosine-5'-Triphosphate Stimulation Do Not Depend on P2X7 Receptor Expression Levels but on Their Activation and Differentiation Stage. Front. Immun., Frontiers.; 9, 360, (2018).

7. Di Virgilio, F., G. Schmalzing, and F. Markwardt, The elusive P2X7 macropore. T Tren. Cell. Bio., Elsevier. 28, 392, (2018).

8. Burnstock, G., Purinergic signalling: therapeutic developments Frontiers in pharmacology. Frontiers.; 8, 661, (2017).

9. Zhang, W., et al., miR-373 regulates inflammatory cytokine-mediated chondrocyte proliferation in osteoarthritis by targeting the $\mathrm{P} 2 \mathrm{X} 7$ receptor. FEBS Open Bio, Wiley Online Library. 8, 325, (2018).

10. Ou, A., B.J. Gu, and J.S. Wiley, The scavenger activity of the human P2X7 receptor differs from $\mathrm{P} 2 \mathrm{X} 7$ pore function by insensitivity to antagonists, 
genetic variation and sodium concentration. Relevance to inflammatory brain diseases Biochimica et Biophysica Acta (BBA)-Molecular Basis of Disease, Elsevier. 1864, 1051, (2018).

11. Zhou, Y., et al., Infection, Genetics and Evolution. Elsevier. 57, 138, (2018).

12. Wei, L., et al., ATP-activated P2X7 receptor in the pathophysiology of mood disorders and as an emerging target for the development of novel antidepressant therapeutics. Neurosci Biobehav R, Elsevier. 87, 192 (2018).

13. Faria, R., et al., 1, 4-Naphthoquinones potently inhibiting $\mathrm{P} 2 \mathrm{X} 7$ receptor activity. Eur J Med Chem.143, 1361, (2 018).

14. Leyva, E., et al., Importancia quimica y biológica de naftoquinonas. evisión bibliográfica. Afinidad.; 74 (2017).

15. Wiley, J., et al., The human $\mathrm{P} 2 \mathrm{X} 7$ receptor and its role in innate immunity. HLA. 78, 321, (2011.)

16. Liu, C.S., G.-N.; Luo, Y.-H.; Piao, X.-J.; Jiang, X.-Y.; Meng, L.-Q. Wang, Y.; Zhang, Y.; Wang, J.-R.; Wang, Novel 1, 4-Naphthoquinone derivatives induce apoptosis via ROS-mediated p38/MAPK, Akt and STAT3 signaling in human hepatoma Hep3B cells Article (liu2018novel) Liu, C.; Shen, G.-N.; Luo, Y.-H.; Piao, X.-J.; Jiang, X.-Y.; Meng, L.-Q.; Wang, Y.; Zhang, Y.; Wang, J.-R.; Wang, H. \& others Novel 1, 4-Naphthoquinone derivatives induce apoptosis via ROS-mediated p38/MAPK, Akt and STAT3 signaling in human hepatoma Hep3B cells. The international J. Biochem. \& cell biology, Elsevier, (2018).

17. Sandur, S.K., et al., Plumbagin (5-hydroxy-2-methyl-1, 4-naphthoquinone) suppresses NF- $\kappa$ B activation and NF- $\kappa$ B-regulated gene products through modulation of $\mathrm{p} 65$ and $\mathrm{I} \kappa \mathrm{B} \alpha$ kinase activation, leading to potentiation of apoptosis induced by cytokine and chemotherapeutic agents. J. Bio. Chem.,. 281, 7023, (2006).

18. Lara, L.M., C.; Calvet, C.; Lechuga, G.; Souza, R.; Bourguignon, S.; Ferreira, V.; Rocha, D. \& Pereira, M, Efficacy of 2-hydroxy-3-phenylsulfanylmethyl-[1, 4]-naphthoquinone derivatives against different Trypanosoma cruzi discrete type units: Identification of a promising hit compound European J Med. Chem., Elsevier, 144, 572, (2018).

19. Bao, N.O., J. Shi, W. Li, N. Chen, L. Sun, J., Highly Efficient Synthesis and Structure--Activity Relationships of a Small Library of Substituted 1 , 4-Naphthoquinones. Eur J Med Chem, Wiley Online Library, 2018, 2254, (2018).

20. Alarcón, D., F. Gatica-Díaz, and J.S. Gómez-Jeria, Modeling the relationships between molecular structure and inhibition of virus-induced cytophatic effects. Anti-HIV and anti-H1N1 (Influenza) activities as examples. J. Chil. Chem. Soc, 2013.

21. Barahona, C.N., S. Gómez, J, Model-basedquantum-chemical study of the uptake of some polychlorinated pollutant compounds by Zucchini subspecies. J. C. Chem. Soc, , 57, 1497, (2012).

22. Lozano-Aponte, J. and T. Scior, ¿ Qué sabe Ud. acerca de... QSAR? Revista mexicana de ciencias farmacéuticas. 43, 82, (2012).

23. Gómez, J., D. Alarcón, and A. Paz de la Vega, Quantum chemical study of the relationships between electronic structure and pharmacokinetic profile, inhibitory strength toward hepatitis c virus ns $5 \mathrm{~b}$ polymerase and hcv replicons of indole-based compounds. J. C. Chem. Soc. 58, 1842, (2013).

24. Hudson, R. and G. Klopman, A general perturbation treatment of chemical reactivity. Tetrahedron Letters. 8; 1103, (1967).

25. Kooyman, E.C. and J.W. Heringa, Extension of the K-Region Hypothesis of Carcinogenic Chemical. Compounds. Nature, 170, 661, (1952).

26. Gómez-Jeria, J.S., A new set of local reactivity indices within the HartreeFock-Roothaan and density functional theory. Can. Chem. Trans.. 1, 25, (2013).

27. Peradejordi, F., A.N. Martin, and A. Cammarata, Quantum chemical approach to structure-activity relationships of tetracycline antibiotics. J. Pharm. Sci. 60, 576, (1971)

28. Roothaan, C.C., New Developments in Molecular Orbital Theory. Rev. Mod. Phys. 23, 69, (1951).

29. De La Vega A, P., D.A. Alarcon, and J.S. Gómez Jeria, Quantum chemica study of the relationships between electronic structure and pharmacokinetic profile, inhibitory strength toward hepatitis $\mathrm{c}$ virus ns $5 \mathrm{~b}$ polymerase and hev replicons. J. C. Chem. Soc.. 58, 2157, (2013).

30. Zerner, M., Perspective on "New developments in molecular orbital theory". Theor. Chem. Acc.. 103, 217, (2000).

31. Gomez-Jeria, J.S., On some problems in quantum pharmacology I. The partition functions. International journal of quantum chemistry, Wiley Online Library. 23, 1969, (1983).

32. Karelson, M., V. Lobanov, and A. Katritzky, Quantum-chemical descriptors in QSAR/QSPR studies. Chemical reviews. 96, 1027, (1996).
33. Gómez, S.S., Francisco, A Theoretical Study of the Relationships between Electronic Structure and CB1 and CB2 Cannabinoid Receptor Binding Affinity in a Group of 1-Aryl-5-(1-H-pyrrol-1-yl)-1-H-pyrazole-3-carboxamides. J Quan. Chem. 1, (2014).

34. Fukui, K.Y., T. Shingu, H., Molecular Orbital Theory of Reactivity in Aromatic Hydrocarbons. J. Chem. Phys. 20, 722, (1952).

35. Mulliken, R.S., Electronic Population Analysis on LCAO [Single Bond] MO Molecular Wave Functions. I. J. Chem. Phys, 23, 1833, (1955).

36. Gómez Jeria, J., S, P. Castro-Latorre, and G. Kpotin, Quantum Chemical Study of the Relationships between Electronic Structure and Antiviral Activities against Influenza A H1N1, Enterovirus 71 and Coxsackie B3 viruses of some Pyrazine-1, 3-thiazine Hybrid Analogues. International J. R. App., N. and Soc. Sci.; 9, 49, (2017).

37. Sebaugh, J., Guidelines for accurate EC50/IC50 estimation. Pharmaceutical statistics, Wiley Online Library.10, 128, (2011).

38. Gómez-Jeria, J., D-Cent-QSAR: A program to generate Local Atomic Reactivity Indices from Gaussian 03 log files. v. 1.0. Chile, 2014.

39. Solís, R.G., J., A Density Functional Theory study of therelationships between electronic structure and metabotropic glutamate receptor subtype 5 affinity of 2-amino- and 2-halothiazole derivatives. Research J. Pharm, Bio. and Chem. Sci, 5, 1401, (2014).

40. Gómez-Jeria, J.-S.S.L., M. \& Robles N, A, A Density Functional Study of the Inhibition of Microsomal Prostaglandin E2 Synthase-1 by 2-aryl substituted quinazolin-4(3H)-one, pyrido[4,3-d]pyrimidin-4(3H)-one and pyrido[2,3-d]pyrimidin-4(3H)-one derivatives. Der Pharmacia Lettre. 7, 54, (2015).

41. Gómez, J.A., J., A Theoretical Study of the Relationships between Electronic Structure and Receptor Affinity in a group 5-HT1A and 5-HT2A of ligands containing an isonicotinic nucleus. Chem R J. 2, 198, (2017)

42. Gomez Jeria, J.S. and A. Robles Navarro, A theoretical study of the relationships between electronic structure and inhibition of tumor necrosis factor by cyclopentenone oximes. Research j. phar. Bio. and chem. Sci., rjpbcs research j. pharm., bio. \& chem. Sci. rjpbcs research j. pharm., bio. \& chem. Sci., proddatur. 6, 1337, (2015).

43. Gómez Jeria, J., S and G. Kpotin, Some remarks on the interpretation of the local atomic reactivity indices within the Klopman-Peradejordi-Gómez (KPG) method. Research J. Pharm, Bio. and Chem. Sci. 9, 550, (2018).

44. K. Fukui, T.Y., C. Nagata y H. Shingu,, Molecular Orbital Theory of Orientation in Aromatic, Heteroaromatic, and Other Conjugated Molecules. The J Chem Ph,. 22, 1433, (1954).

45. Gomez Jeria, J., S, A Theoretical Study of the Relationships between Electronic Structure and Antifungal Activity against Botrytis cinerea and Colletotrichum lagenarium of a Group of Carabrone Hydrazone Derivatives. Research J. Pharm, Bio. and Chem Sci., 6, 688, (2015)

46. Gómez-Jeria, J.-S.S.-G., R., A Density Functional Theory study of the relationships between electronic structure and metabotropic glutamate receptor subtype 5 affinity of 2-amino- and 2-halothiazole derivatives. J. Pharm, Bio. and Chem. Sci. 5, 1401, (2014).

47. Geerlings, P.D.P., F. \& Langenaeker, W., Conceptual density functional theory. Chemical reviews, ACS Publications. 103, 1793, (2003).

48. Gatica Diaz, J. and J.S. Gómez Jeria, A Theoretical Study of the Relationships between Electronic Structure and Cytotoxicity of a group of N2-alkylated Quaternary $\beta$-Carbolines against nine Tumoral Cell Lines. Comput. Methods Drug Des. 4, 79, (2014)

49. Gómez, J.M.G., Quantum-chemical study of the relationships between electronic structure and anti influenza activity. 1. The inhibition of cytophatic effects produced by the influenza A/Guangdong Luohu/219/2006 (H1N1) strain in MDCK cells by substituted bisaryl amide compounds. j comput aid mol des. 4, 34, (2014).

50. Gómez, J.K., G. K.; , H.-K.; , A. Mensah, J., A DFT study or the relationship between the electronic structure and the antiplasmodial activity of a series of 4-anilino-2-trichloromethylquinazolines derivatives. World Scientific News. 88, 138, (2017).

51. Gómez, J.G., V., A quantum chemical analysis of the inhibition of $\alpha$-glucosidase by a group of oxadiazole benzohydrazone derivatives. Der Pharma Chemic, 2016. 8: p. 2016.

52. Gómez, J.L., M. Robles, N., A Density Functional Study of the Inhibition of Microsomal Prostaglandin E2 Synthase-1 by 2-aryl substituted quinazolin-4(3H)-one, pyrido[4,3-d]pyrimidin-4(3H)-one and pyrido[2,3-d] pyrimidin-4(3H)-one derivatives. Der Pharmacia Lettre. 7, 54, (2105)

53. Gómez, J.R.N., A DFT study of the Inhibition of Carbonic Anhydrase Isoforms I, II, IX and XII by a Series of Benzenesulfonamides and Tetrafluorobenzenesulfonamides. AM J Chem and App. 2, 66, (2015) 